\title{
Resveratrol alleviates Staphylococcus aureus pneumonia by inhibition of the NLRP3 inflammasome
}

\author{
SUXIA WU ${ }^{1}$ and JIANAN HUANG ${ }^{2}$ \\ ${ }^{1}$ Department of Respiratory Medicine, Zhangjiagang City Jingfeng People's Hospital, Suzhou, Jiangsu 215625; ${ }^{2}$ Department of \\ Respiratory Medicine, The First Affiliated Hospital of Soochow University, Suzhou, Jiangsu 215006, P.R. China
}

Received July 8, 2016; Accepted April 10, 2017

DOI: $10.3892 /$ etm.2017.5337

\begin{abstract}
Staphylococcus aureus (S. aureus) causes a wide variety of infections, including $S$. aureus pneumonia. Resveratrol, a natural polyphenolic compound contained in several plant species, exerts various activities, including anti-bacterial and pulmonary protective activities. The aim of the present study was to evaluate the protective effect of resveratrol on a murine model of $S$. aureus pneumonia and to elucidate the underlying mechanisms. It was found that resveratrol significantly reduced $S$. aureus-induced mortality, ameliorated lung injury and decreased cytokine levels in the bronchoalveolar lavage fluid and lung tissue of $S$. aureus infected-mice. In addition, reverse-transcription quantitative polymerase chain reaction and western blot assays showed that resveratrol markedly decreased the mRNA and protein expression of nucleotide-binding domain and leucine-rich repeat containing gene family pyrin domain containing 3 protein (NLRP3), apoptosis-associated speck-like protein containing a $\mathrm{C}$-terminal caspase recruitment domain and caspase-1. These results demonstrated that resveratrol significantly alleviates S. aureus pneumonia in mice, the possible underlying mechanism of which is associated with the inhibition of the NLRP3 inflammasome. The present study suggested that resveratrol is a potentially useful agent for the treatment of $S$. aureus pneumonia and $S$. aureus-induced infectious diseases.
\end{abstract}

\section{Introduction}

Staphylococcus aureus (S. aureus) is an important Gram-positive human pathogen that causes various diseases, including typical skin and soft-tissue infections, as well as life-threatening invasive diseases (1). S. aureus pneumonia,

Correspondence to: Dr Suxia Wu, Department of Respiratory Medicine, Zhangjiagang City Jingfeng People's Hospital, 35 Jingzhong Road, Jingfeng Town, Zhangjiagang, Suzhou, Jiangsu 215625, P.R. China

E-mail: sxw2156@163.com

Key words: Staphylococcus aureus pneumonia, resveratrol, NLRP3 inflammasome, IL-1 $\beta$, inflammation characterized by localized necrosis and inflammation, is one of the most prevalent $S$. aureus-mediated diseases and occurs in $\sim 13.3 \%$ of all invasive staphylococcal infections $(2,3)$. However, due to the ability of $S$. aureus to develop resistance to a broad range of antibiotics, a decline in the efficiency of current treatments has been observed, and there is an obvious requirement for the development of novel antibiotics or therapeutic strategies against staphylococcal infections.

Recently, the innate immune pathway involving the inflammasome was demonstrated to have a key role in several diseases, including atherosclerosis (4), Alzheimer's disease (5) and type 2 diabetes (6). The nucleotide-binding domain and leucine-rich repeat containing gene (NLR) family, pyrin domain containing 3 protein (NLRP3) inflammasome consists of the NLR family member NLRP3, the adaptor protein apoptosis-associated speck-like protein containing a C-terminal caspase recruitment domain (ASC) and the effector protein caspase-1 (7). The NLRP3 inflammasome has been demonstrated to be activated in response to a broad range of exogenous and endogenous stimuli, including infecting microorganisms such as Sendai virus (8), adenovirus (9) and Candida albicans (10), as well as S. aureus (11), and Shigella flexneri (12). Furthermore, $S$. aureus $\alpha$-hemolysin was demonstrated to induce interleukin (IL) $-1 \beta$ secretion and caspase- 1 activation in human and mouse monocytic cells through the NLRP3 inflammasome (13). Furthermore, the activation of NLRP3 was found to have a key role in a murine model of severe pneumonia induced by $S$. aureus $\alpha$-hemolysin (14), suggesting the NLRP3 inflammasome as a potential target for pharmacologic interventions in severe $S$. aureus pneumonia.

Resveratrol (3,5,4'-trihydroxystilbene or 3,5,4'-stilbenetriol) is a natural polyphenolic compound contained in several plant species, such as grapes, berries and peanuts (15). Resveratrol has been shown to have various activities, including an-inflammatory (16), anti-bacterial (17) and anti-oxidant effects (18). Furthermore, resveratrol has also been demonstrated to exert an anti-inflammatory effect on $S$. aureus-induced keratitis by decreasing cell surface Toll-like receptor 2 and downregulating the expression of IL-8 (19). A previous study found that resveratrol protects $S$. aureus-induced lung inflammation in human lung epithelial cells (20). These findings suggested that resveratrol may be effective in ameliorating $S$. aureus pneumonia. In addition, numerous studies have indicated that resveratrol inhibits the activation of NLRP3-mediated 
inflammation in serious diseases, such as myocardial ischemia/reperfusion injury (21), metaflammation (22) and chronic kidney disease (23). Thus, in the present study, it was hypothesized that resveratrol ameliorates $S$. aureus-induced pneumonia. Furthermore, it was investigated whether resveratrol exerts this effect via inhibition of the activation of the NLRP3 inflammasome pathway.

\section{Materials and methods}

Reagents and bacteria. S. aureus 8325-4 and DU 1090 were purchased from Wenzhou Kont Biology \& Technology Co. Ltd (Jiangsu, China). S. aureus strains were cultured in tryptone soya broth (Oxoid, Basingstoke, UK). TNF- $\alpha$ (438208), IL-1 $\beta$ (437008) and IL-18 (437008) ELISA kits were purchased from BioLegend Inc. (San Diego, CA, USA). TRIzol solution, a reverse-transcription-polymerase chain reaction (RT-PCR) kit and a fluorescence quantitative PCR kit were purchased from Takara Bio Inc. (Otsu, Japan). Antibodies against NLRP3 (13158) and ASC (67824) were purchased from Cell Signaling Technology, Inc. (Danvers, MA, USA). Antibodies against caspase-1 (sc-514) and GAPDH (sc-25778) were purchased from Santa Cruz Biotechnology, Inc. (Dallas, TX, USA). Horseradish peroxidase-conjugated anti-rabbit immunoglobulin G antibody (074-1506) was purchased from KPL (Gaithersburg, MD, USA). Resveratrol, purchased from Sigma-Aldrich (Merck KGaA, Darmstadt, Germany), was suspended in PBS for the animal studies.

Mouse model of S. aureus pneumonia. A total of 93 female C57BL/6J mice (age, 8 weeks; weight, 18-22 g) were purchased from the Experimental Animal Center of Soochow University (Suzhou, China). Mice were divided into three groups: S. aureus + PBS group, S. aureus + resveratrol group and DU $1090+$ PBS group. The DU 1090 group was designed as the comparative group for $S$. aureus infected group treated with PBS. After anesthesia with $80 \mathrm{mg} / \mathrm{kg}$ ketamine and $15 \mathrm{mg} / \mathrm{kg}$ xylazine (Hubei Xinkang Pharmaceutical Chemical Co., Ltd; intraperitoneally) (24), S. aureus pneumonia was induced by addition of $30 \mu \mathrm{l}$ of $S$. aureus suspension (4x10 ${ }^{8}$ CFUs, $S$. aureus $8325-4$, Wenzhou Kont Biology \& Technology Co., Ltd., Jiangsu, China) into the left nostril. Mice in the DU $1090+$ PBS group were intranasally infected with $30 \mu 1$ DU 1090 (4x108 CFUs; Wenzhou Kont Biology \& Technology Co., Ltd.). The infected mice were subcutaneously administered PBS or $30 \mathrm{mg} / \mathrm{kg}$ resveratrol $2 \mathrm{~h}$ after infection and then at $12 \mathrm{~h}$ intervals. The mice were housed at $30^{\circ} \mathrm{C}$ with $45-75 \%$ humidity. Mice had free access to food and water with a $12 \mathrm{~h} \mathrm{light/dark} \mathrm{cycle.} \mathrm{The} \mathrm{mortality}$ was monitored over a 72-h time course.

All animal studies were performed according to the experimental practices and standards developed by the Animal Welfare and Research Ethics Committee of Zhangjiagang City Jingfeng People's Hospital (Suzhou). The experimental protocols were approved by the animal care committee of Zhangjiagang City Jingfeng People's Hospital (Suzhou).

Hematoxylin and eosin (H\&E) staining analysis. Lungs were removed and fixed at room temperature in $10 \%$ formalin for one day. Formalin-fixed tissues were processed, paraffin-embedded, thin sectioned ( $5 \mu \mathrm{m})$, stained with $\mathrm{H} \& \mathrm{E}$ (hematoxylin for $5 \mathrm{~min}$ and eosin for $20 \mathrm{sec}$ at room temperature) and visualized by light microscopy (Olympus, Tokyo, Japan). A total of 9 mice were used for H\&E analysis.

ELISA. Bronchoalveolar lavage fluid (BALF) collection was performed twice by intratracheal instillation of $500 \mu 1$ PBS. The lung tissues were then harvested and homogenized with PBS. After centrifugation $\left(1,500 \mathrm{x} \mathrm{g}, 10 \mathrm{~min}, 4^{\circ} \mathrm{C}\right)$, the supernatants of BALF and lung tissue lysates were individually used for cytokine measurements. Cytokine levels were measured using ELISA kits for interleukin (IL)-1 $\beta$, IL-18 and tumor necrosis factor (TNF)- $\alpha$.

$R T$ quantitative (q)PCR. Total RNA of lung tissues was isolated using TRIzol (Takara Bio, Inc.) and DNA was removed from each RNA preparation using RNase-free DNase I (Takara Bio Inc.) according to the manufacturer's instructions. For RT, total RNA $(1 \mu \mathrm{g})$ was reverse transcribed to first-strand cDNA in $20 \mu \mathrm{l}$ of mixture containing $25 \mathrm{mM}$ $\mathrm{MgCl}_{2}(4 \mu \mathrm{l})$, reverse transcription 10X buffer $(2 \mu \mathrm{l}), 10 \mathrm{mM}$ dNTP mixture $(2 \mu \mathrm{l})$, recombinant RNase inhibitor $(0.5 \mu \mathrm{l})$, AMV reverse transcriptase (15 $\mu 1$ and Oligo (dT) primers (0.5 $\mu \mathrm{g}$; RNA PCR kit; cat. no. RR019B; Takara Bio, Inc.). The reaction conditions were as follows: $42^{\circ} \mathrm{C}$ for $60 \mathrm{~min}$ followed by $95^{\circ} \mathrm{C}$ for $5 \mathrm{~min}$. qPCR was performed using a 7000 Sequence Detection System (Applied Biosystems; Thermo Fisher Scientific, Inc., Waltham, MA, USA) using SYBR Premix Ex Taq (Takara Bio, Inc.) according to the manufacturer's protocol. GAPDH is used as an internal control. The PCR reaction system contained $2 \mu \mathrm{g}$ template cDNA, ligase, dNTP, buffer 10x buffer, $200 \mu \mathrm{mol} / 1$ of each dNTP, $20 \mathrm{pmol}$ of each primer, $1.5 \mathrm{mmol} / 1 \mathrm{Mg} 2+$ and nuclease-free water. PCR was performed using the following program: $94^{\circ} \mathrm{C}$ for $5 \mathrm{~min}$, followed by 30 cycles of $94^{\circ} \mathrm{C}$ for $30 \mathrm{sec}, 58^{\circ} \mathrm{C}$ for $60 \mathrm{sec}$ and $72^{\circ} \mathrm{C}$ for $45 \mathrm{sec}$. Primers used in this study are listed in Table I. The relative expressions were calculated using the $2^{-\Delta \Delta \mathrm{Cq}}$ method (25).

Western blot analysis. Total protein extraction of mouse lung tissues was performed for western blot analysis using lysis buffer (10 mM Tris-HCl, $1 \mathrm{mM}$ EDTA, and $250 \mathrm{mM}$ sucrose, $\mathrm{pH} 7.4$, containing $15 \mu \mathrm{g} / \mathrm{ml}$ aprotinin, $5 \mu \mathrm{g} / \mathrm{ml}$ leupeptin, $0.1 \mathrm{mM}$ phenylmethanesulfonyl fluoride, $1 \mathrm{mM} \mathrm{NaF}$ and $1 \mathrm{mM} \mathrm{Na} \mathrm{VO}_{4}$ ). A total of $20 \mathrm{mg}$ protein was separated $12 \%$ SDS-PAGE and transferred onto polyvinylidene fluoride membranes (Millipore, Billerica, MA, USA). Membranes were incubated with primary antibodies, including antibodies against NLRP3 (1:1,000 dilution), ASC (1:1,000 dilution), Caspase-1 (1:300 dilution), pro-Caspase-1 (1:300 dilution, ERP16883; Abcam, Cambridge, MA, USA) and GAPDH (1:500 dilution) overnight at $4^{\circ} \mathrm{C}$. Subsequent to secondary antibody incubation with horseradish peroxidase-conjugated goat anti-rabbit IgG H\&L (1:10,000; ab97051; Abcam) for $2 \mathrm{~h}$ at room temperature, immunoreactive bands were visualized by incubation with lumiGLO reagent (Cell Signaling Technologies, Inc.) and exposed to X-ray film according to the manufacturer's instructions. Relative quantitation of western blots was performed with normalization to the amount of GAPDH protein levels. 
Table I. Primer sequences used for polymerase chain reaction in the present study.

\begin{tabular}{lll}
\hline Gene & \multicolumn{1}{c}{ Forward } & \multicolumn{1}{c}{ Reverse } \\
\hline NLRP3 & 5'-GGTCCTCTTTACCATGTGTGCTTC-3' & 5'-AAGTCATGTGGCTGAAGCTGTA-3' \\
ASC & 5'-CTTGTCAGGGGATGAACTCAA-3' & 5'-CTGGTCCACAAAGTGTCCTGT-3' \\
Caspase-1 & 5'-CGTGGAGAGAAACAAGGAGTG-3' & 5'-AATGAAAAGTGAGCCCCTGAC-3' \\
GAPDH & 5'-AGGTCGGTGTGAACGGATTTG-3' & 5'-GGGGTCGTTGATGGCAACA-3'
\end{tabular}

NLRP3, NLR family pyrin domain containing 3 protein; ASC, apoptosis-associated speck-like protein containing a C-terminal caspase recruitment domain.

Statistical analysis. The experimental data were assessed using an independent Student's t-test with SPSS 13.0 statistical software (SPSS, Inc., Chicago, IL, USA). P<0.05 was considered to indicate a statistically significant difference.

\section{Results}

Resveratrol protects mice from S. aureus infection. To evaluate the effectiveness of resveratrol on $S$. aureus pneumonia, mice were intranasally administered $S$. aureus at a lethal dose. As displayed in Fig. 1, the mortality rate of $S$. aureus-infected mice treated with resveratrol was significantly lower than that of those treated with PBS $(\mathrm{P}<0.05$ at 24 and $72 \mathrm{~h}$ and $\mathrm{P}<0.01$ at $48 \mathrm{~h}$ ). These results suggested that resveratrol protects against S. aureus infection at a lethal dose.

Resveratrol alleviates $S$. aureus pneumonia in mice. To investigate the effect of resveratrol treatment on pneumonia-associated lung injury and pathological changes, histopathological analysis of the lungs was performed at $24 \mathrm{~h}$ after infection. H\&E staining demonstrated that $S$. aureus infection induced severe alveolar destruction and massive inflammatory cell aggregation in the lung tissue (Fig. 2A), while treatment with resveratrol obviously reduced these changes in the lung tissue (Fig. 2B). These results suggested that resveratrol alleviated $S$. aureus pneumonia in mice.

Resveratrol decreases inflammatory cytokine production in $S$. aureus pneumonia. The present study then investigated the secretion of inflammatory cytokines in the BALF and lung tissues of mice infected with $S$. aureus. The mean value of IL-1 $\beta$, IL-18 and TNF- $\alpha$ expression in BALF and lung tissues in two $S$. aureus and DU 1090 groups are as follows: IL-1 $\beta$ (BALF: 83.062, 49.332, 28.404, lung: 166.123, 98.664, 56.808, IL-18 (BALF: 222.02, 160.694, 33.09, lung: 444.042, 321.386, 66.183) and TNF- $\alpha$ (BALF: 98.083, 70.325, 29.223, lung: $196.165,140.651,58.447)$. As illustrated in Fig. 3, $S$. aureus significantly increased the levels of IL-1 $\beta$, IL-18 and TNF- $\alpha$ in the BALF. Compared to the model group, mice treated with resveratrol showed a lower level of IL-1 $\beta$ $(\mathrm{P}<0.01)$, IL-18 $(\mathrm{P}<0.01)$ and TNF- $\alpha(\mathrm{P}<0.01)$ in the BALF. Similar results were also observed in the lung tissues (Fig. 3D-F). These results indicated that resveratrol treatment decreases the inflammatory response by reducing the production of inflammatory cytokines in mice with $S$. aureus pneumonia.

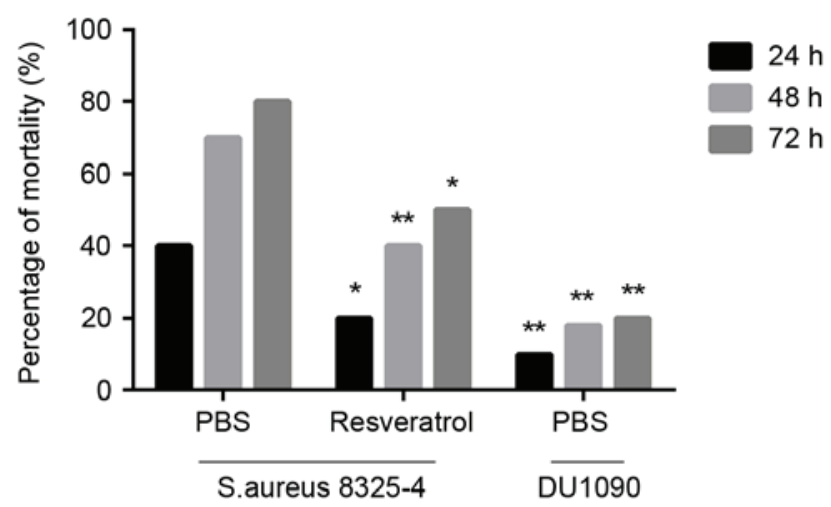

Figure 1. Effect of resveratrol on the mortality rate of mice infected with S. aureus strains. Each group contained 20 mice. ${ }^{*} \mathrm{P}<0.05,{ }^{* *} \mathrm{P}<0.01$ vs. mice infected with $S$. aureus 8325-4 after treatment with PBS. S. aureus, Staphylococcus aureus.

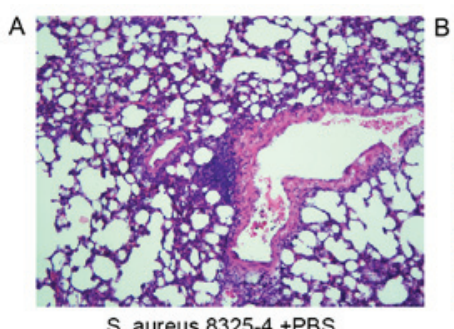

S. aureus $8325-4+$ PBS

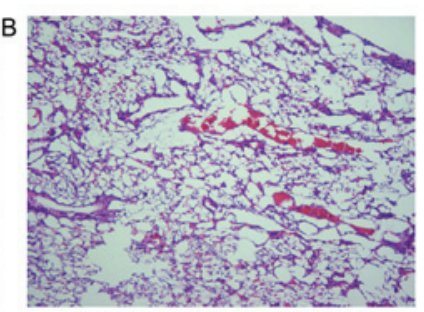

S. aureus $8325-4+$ resveratrol

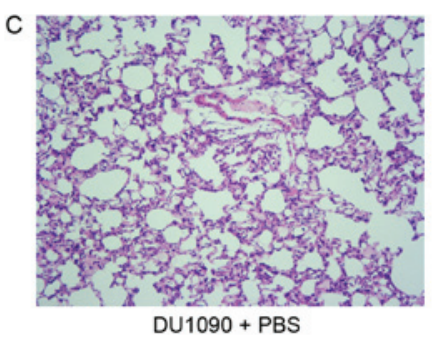

Figure 2. Effect of resveratrol on the histopathology of S. aureus-infected lung tissue of mice. Sections from mice infected with (A) S. aureus 8325-4 after treatment with PBS, (B) S. aureus 8325-4 after treatment with resveratrol and (C) DU 1090 after treatment with PBS were stained with hematoxylin and eosin. Magnification, x200. S. aureus, Staphylococcus aureus.

Resveratrol inhibits the activation of the NLRP3 inflammasome in S. aureus-infected mice. To analyze the impact of resveratrol on the NLRP3 inflammasome in the lungs of infected mice, RT-qPCR and western blot analyses were 
A

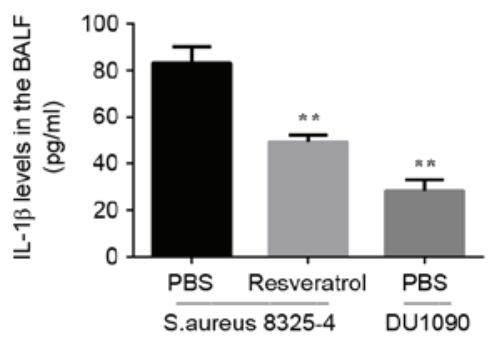

D

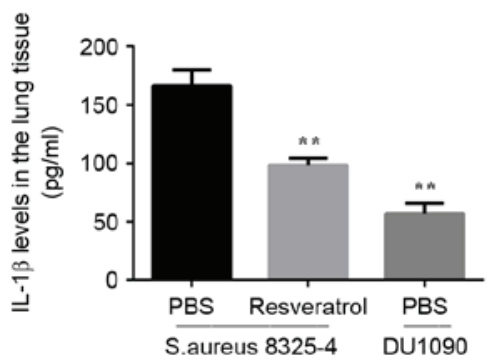

B
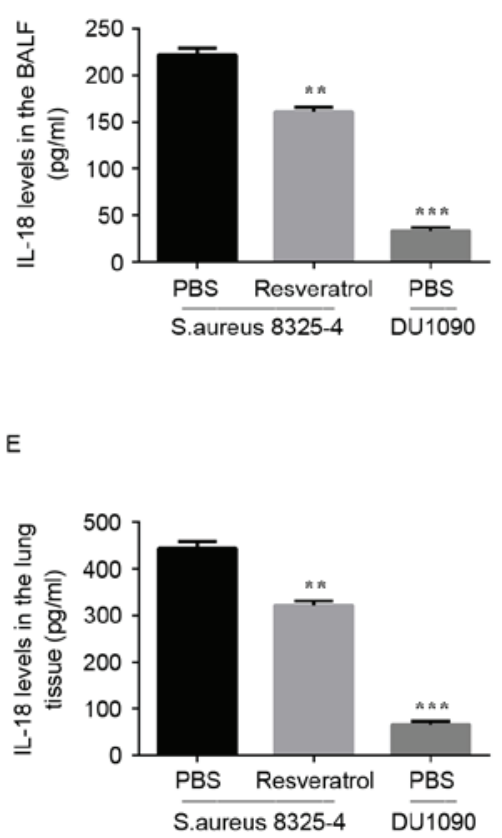

C

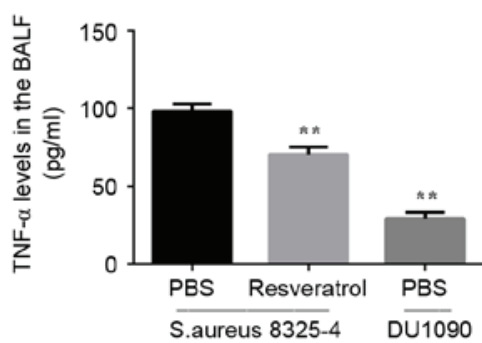

$\mathrm{F}$

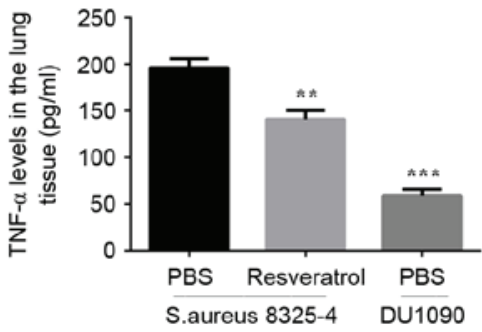

Figure 3. Effect of resveratrol on cytokine production in mice infected with S. aureus. Cytokine concentrations in (A-C) the BALF and (D-F) lung issue were measured by ELISA kits for IL-1 $\beta$, IL-18 and TNF- $\alpha$ according to the manufacturer's instructions. Results are expressed as the mean \pm standard deviation of three independent experiments. ${ }^{* *} \mathrm{P}<0.01 ;{ }^{* * *} \mathrm{P}<0.001$ vs. mice infected with $S$. aureus $8325-4$ after treatment with PBS. BALF, bronchoalveolar lavage fluid; IL, interleukin; TNF, tumor necrosis factor; S. aureus, Staphylococcus aureus.

A

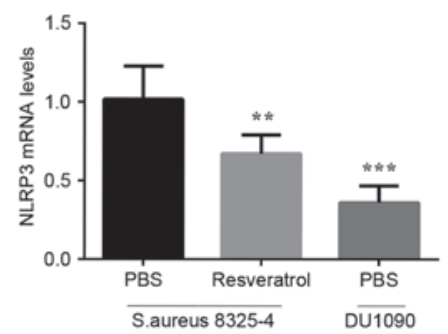

D

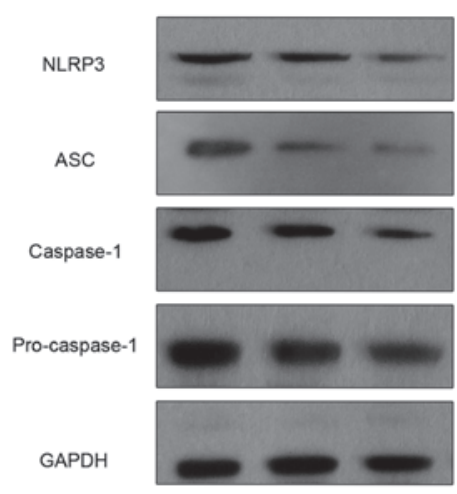

B

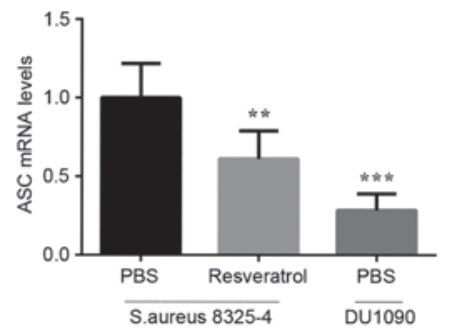

E
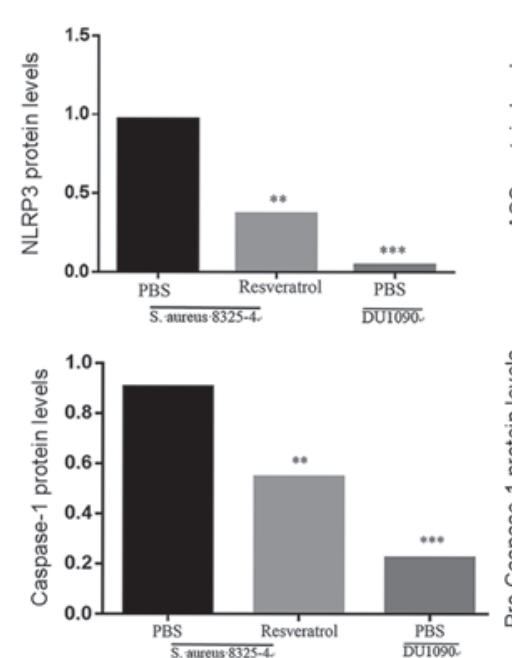

$\mathrm{C}$
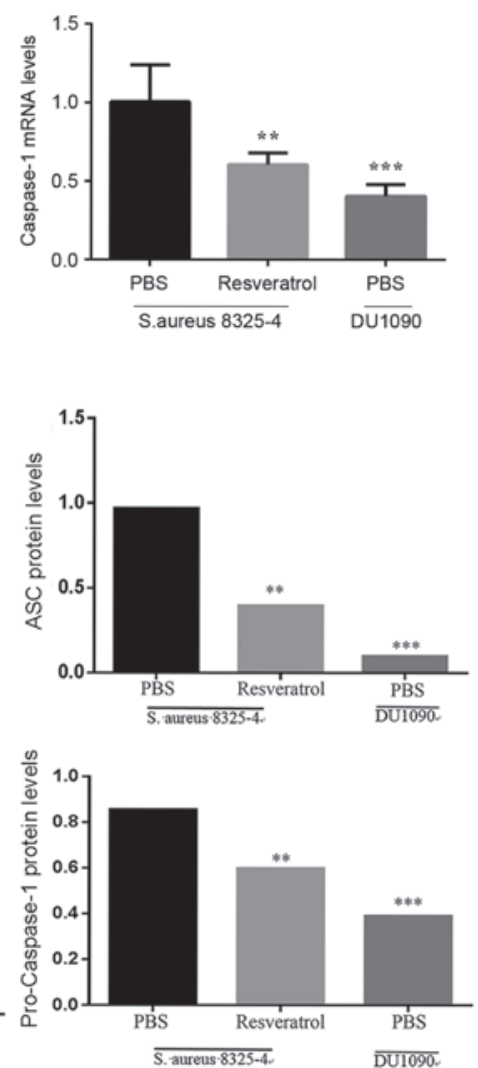

Figure 4. Effect of resveratrol on the expression of NLRP3 inflammasome in mice infected with S. aureus. Mice were treated with resveratrol for $24 \mathrm{~h}$ prior to S. aureus infection. The (A-C) RNA and (D) protein expression of NLRP3, ASC and caspase-1 were measured by reverse-transcription quantitative polymerase chain reaction and western blot. (E) Quantified western blotting results. Values are expressed as the mean \pm standard deviation. ${ }^{* *} \mathrm{P}<0.01$; ${ }^{* * *} \mathrm{P}<0.001$ vs. mice infected with S. aureus 8325-4 after the treatment of PBS. NLRP3, nucleotide-binding domain and leucine-rich repeat containing gene family pyrin domain containing 3 protein; ASC, apoptosis-associated speck-like protein containing a C-terminal caspase recruitment domain; S. aureus, Staphylococcus aureus. 
used to estimate their expression in lungs of pneumonia model mice at $24 \mathrm{~h}$ after $S$. aureus infection. As illustrated in Fig. 4A-C, the mRNA expression levels of NLRP3, ASC and caspase-1 in the lungs were obviously increased in $S$. aureus-infected mice in comparison with mice in the DU 1090 group indicating the activation of the NLRP3 inflammasome. Furthermore treatment with resveratrol significantly decreased the mRNA levels of NLRP3, ASC and caspase- 1 in this animal model. In addition, western blot analysis revealed that $S$. aureus significantly increased the protein levels of NLRP3 and ASC compared with the PBS treated DU 1090 infected group, and stimulated the activation of caspase-1 (Fig. 4D), which was attenuated by the treatment with resveratrol. These results demonstrated that resveratrol inhibited the activation of the NLRP3 inflammasome in the lungs of $S$. aureus-infected mice.

\section{Discussion}

To the best of our knowledge, the present study was the first to demonstrate that resveratrol exerts a protective effect against lung injury in a murine model of $S$. aureus pneumonia, and that the effect is probably associated with the reduction of the inflammatory response and inhibition of the NLRP3 inflammasome.

Increasing evidence indicated that certain natural products protect mice from $S$. aureus pneumonia (26-28). A previous study demonstrated that resveratrol had obvious anti-bacterial activities against clinical isolates of methicillin-resistant S. aureus (29). Similarly, the present results showed that treatment with resveratrol significantly reduced the mortality in a murine model of $S$. aureus pneumonia. Consistent with a previous study (26), the histopathology results showed that $S$. aureus induced severe alveolar destruction and massive inflammatory cell aggregation in the lung. Of note, resveratrol significantly ameliorated $S$. aureus-induced lung injury in mice, suggesting a protective effect of resveratrol against $S$. aureus pneumonia in mice.

$S$. aureus pneumonia induces a strong host inflammatory reaction, which causes rapid and excessive recruitment of inflammatory cells (30). The present study found that pro-inflammatory cytokines, including IL-1 $\beta$, IL-18 and TNF- $\alpha$, were increased in the BALF and lungs. However, resveratrol obviously reduced the elevated levels of IL-1 $\beta$, IL-18 and TNF- $\alpha$ in the BALF and lungs induced by S.aureus infection in mice. These results suggested that resveratrol may have therapeutic potential by reducing the inflammatory response in mice with $S$. aureus-infected lung tissues.

Several studies reported that the NLRP3 inflammasome has a crucial role in the host immune response to $S$. aureus and may be an important target pathway for the treatment of $S$. aureus pneumonia $(14,31)$. In accordance with this, the present study found that resveratrol treatment decreased the mRNA and protein expression of NLRP3, ASC and caspase-1 in the lung tissue of mice with $S$. aureus pneumonia. As is known, activation of the NLRP3 inflammasome promotes the cleavage and activation of caspase- 1 , which in turn is a central stimulator of the pro-inflammatory cytokines IL-1 $\beta$ and IL-18, resulting in inflammation $(32,33)$; this is consistent with the results of the present study regarding pro-inflammatory cytokine secretion observed in BALF and lung tissues in mice infected with $S$. aureus. Collectively, these results suggested that resveratrol exerts a protective effect against $S$. aureus pneumonia, probably via suppression of the NLRP3 inflammasome. However, further study required to clarify the precise mechanism by which resveratrol inhibits the activation of the NLRP3 inflammasome.

In conclusion, the present study demonstrated that resveratrol alleviated $S$. aureus pneumonia in mice by inhibiting NLRP3-mediated inflammation. The results suggested that resveratrol is a potentially useful agent for the treatment of $S$. aureus pneumonia and associated infectious diseases.

\section{References}

1. Lowy FD: Staphylococcus aureus infections. N Engl J Med 339: 520-532, 1998

2. Klevens RM, Morrison MA, Nadle J, Petit S, Gershman K, Ray S, Harrison LH, Lynfield R, Dumyati G, Townes JM, et al: Invasive methicillin-resistant Staphylococcus aureus infections in the United States. JAMA 298: 1763-1771, 2007.

3. Kitur K and Prince A: Staphylococcus aureus activation of NLRP3 inflammasome and necroptosis through MLKL exacerbates pneumonia. FASEB J 29 (1 Suppl): S148.5, 2015.

4. Duewell P, Kono H, Rayner KJ, Sirois CM, Vladimer G, Bauernfeind FG, Abela GS, Franchi L, Nuñez G, Schnurr M, et al: NLRP3 inflammasomes are required for atherogenesis and activated by cholesterol crystals. Nature 464: 1357-1361, 2010.

5. Heneka MT, Kummer MP, Stutz A, Delekate A, Schwartz S, Vieira-Saecker A, Griep A, Axt D, Remus A, Tzeng TC, et al: NLRP3 is activated in Alzheimer/'s disease and contributes to pathology in APP/PS1 mice. Nature 493: 674-678, 2013.

6. Lee HM, Kim JJ, Kim HJ, Shong M, Ku BJ and Jo EK: Upregulated NLRP3 inflammasome activation in patients with type 2 diabetes. Diabetes 62: 194-204, 2013.

7. Jin $C$ and Flavell RA: Molecular mechanism of NLRP3 inflammasome activation. J Clin Immunol 30: 628-631, 2010.

8. Kanneganti TD, Body-Malapel M, Amer A, Park JH, Whitfield J, Franchi L, Taraporewala ZF, Miller D, Patton JT, Inohara N and Núñez G: Critical role for Cryopyrin/Nalp3 in activation of caspase-1 in response to viral infection and double-stranded RNA. J Biol Chem 281: 36560-36568, 2006.

9. Muruve DA, Périlli V, Zaiss AK, White LR, Clark SA, Ross PJ, Parks RJ and Tschopp J: The inflammasome recognizes cytosolic microbial and host DNA and triggers an innate immune response. Nature 452: 103-107, 2008.

10. Wilson D, Thewes S, Zakikhany K, Fradin C, Albrecht A, Almeida R, Brunke S, Grosse K, Martin R, Mayer F, et al: Identifying infection-associated genes of Candida albicans in the postgenomic era. FEMS Yeast Res 9: 688-700, 2009.

11. Mariathasan S, Weiss DS, Newton K, McBride J, O'Rourke K, Roose-Girma M, Lee WP, Weinrauch Y, Monack DM and Dixit VM: Cryopyrin activates the inflammasome in response to toxins and ATP. Nature 440: 228-232, 2006.

12. Willingham SB, Bergstralh DT, O'Connor W, Morrison AC, Taxman DJ, Duncan JA, Barnoy S, Venkatesan MM, Flavell RA, Deshmukh M, et al: Microbial pathogen-induced necrotic cell death mediated by the inflammasome components CIAS1/cryopyrin/NLRP3 and ASC. Cell Host Microbe 2: 147-159, 2007.

13. Craven RR, Gao X, Allen IC, Gris D, Bubeck Wardenburg J, McElvania-TeKippe E, Ting JP and Duncan JA: Staphylococcus aureus alpha-hemolysin activates the NLRP3-inflammasome in human and mouse monocytic cells. PLoS One 4: e7446, 2009.

14. Kebaier C, Chamberland RR, Allen IC, Gao X, Broglie PM, Hall JD, Jania C, Doerschuk CM, Tilley SL and Duncan JA: Staphylococcus aureus $\alpha$-hemolysin mediates virulence in a murine model of severe pneumonia through activation of the NLRP3 inflammasome. J Infect Dis 205: 807-817, 2012.

15. Chun-Fu W, Jing-Yu Y, Fang W and Xiao-Xiao W: Resveratrol: Botanical origin, pharmacological activity and applications. Chin J Nat Med 11: 1-15, 2013. 
16. Bollmann F, Art J, Henke J, Schrick K, Besche V, Bros M, Li H, Siuda D, Handler N, Bauer F, et al: Resveratrol post-transcriptionally regulates pro-inflammatory gene expression via regulation of KSRP RNA binding activity. Nucleic Acids Res 42: 12555-12569, 2014.

17. Hwang D and Lim YH: Resveratrol antibacterial activity against Escherichia coli is mediated by Z-ring formation inhibition via suppression of FtsZ expression. Sci Rep 5: 10029, 2015.

18. Gülçin İ: Antioxidant properties of resveratrol: A structure-activity insight. Innov Food Sci Emerg Technol 11: 210-218, 2010.

19. Marino A, Santoro G, Spataro F, Lauriano ER, Pergolizzi S, Cimino F, Speciale A, Nostro A, Bisignano G and Dugo G: Resveratrol role in Staphylococcus aureus-induced corneal inflammation. Pathog Dis 68: 61-64, 2013.

20. Lee IT, Lin CC, Hsu CK, Wu MY, Cho RL and Yang CM: Resveratrol inhibits Staphylococcus aureus-induced TLR2/MyD88/NF-кB-dependent VCAM-1 expression in human lung epithelial cells. Clin Sci (Lond) 127: 375-390, 2014.

21. Dong W, Yang R, Yang J, Yang J, Ding J, Wu H and Zhang J: Resveratrol pretreatment protects rat hearts from ischemia/reperfusion injury partly via a NALP3 inflammasome pathway. Int J Clin Exp Patho 8: 8731-8741, 2015.

22. Yang SJ and Lim Y: Resveratrol ameliorates hepatic metaflammation and inhibits NLRP3 inflammasome activation. Metabolism 63: 693-701, 2014.

23. Chang YP, Ka SM, Hsu WH, Chen A, Chao LK, Lin CC, Hsieh CC, Chen MC, Chiu HW, Ho CL, et al: Resveratrol inhibits NLRP3 inflammasome activation by preserving mitochondrial integrity and augmenting autophagy. J Cell Physiol 230: 1567-1579, 2015.

24. Veterinary Anesthetic and Analgesic Formulary. 3rd edition, Version G. University of Colorado Denver, Anschutz Medical Campus, 2012

25. Livak KJ and Schmittgen TD: Analysis of relative gene expression data using real-time quantitative PCR and the 2(-Delta Delta C(T)) method. Methods 25: 402-408, 2001.
26. Zhang Y, Wang JF, Dong J, Wei JY, Wang YN, Dai, XH, Wang X, Luo MJ, Tan W, Deng XM and Niu XD: Inhibition of $\alpha$-toxin production by subinhibitory concentrations of naringenin controls Staphylococcus aureus pneumonia. Fitoterapia 86: 92-99, 2013.

27. Qiu J, Niu X, Dong J, Wang D, Wang J, Li H, Luo M, Li S, Feng H and Deng X: Baicalin protects mice from Staphylococcus aureus pneumonia via inhibition of the cytolytic activity of $\alpha$-hemolysin. J Infect Dis 206: 292-301, 2012.

28. Dong J, Qiu J, Wang J, Li H, Dai X, Zhang Y, Wang X, Tan W, Niu X, Deng $X$ and Zhao S: Apigenin alleviates the symptoms of Staphylococcus aureus pneumonia by inhibiting the production of alpha-hemolysin. FEMS Microbiol Lett 338: 124-131, 2013.

29. Su Y, Ma L, Wen Y, Wang H and Zhang S: Studies of the in vitro antibacterial activities of several polyphenols against clinical isolates of methicillin-resistant Staphylococcus aureus. Molecules 19: 12630-12639, 2014.

30. Zhao G, Wu H, Jiang K, Rui G, Zhu Z, Qiu C, Guo M and Deng G: IFN- $\tau$ inhibits $S$. aureus-induced inflammation by suppressing the activation of NF- $\kappa$ B and MAPKs in RAW 264.7 cells and mice with pneumonia. Int Immunopharmacol 35: 332-340, 2016.

31. Muñoz-Planillo R, Franchi L, Miller LS and Núñez G: A critical role for hemolysins and bacterial lipoproteins in Staphylococcus aureus-induced activation of the Nlrp3 inflammasome. J Immunol 183: 3942-3948, 2009.

32. Netea MG, Nold-Petry CA, Nold MF, Joosten LA, Opitz B, van der Meer JH, van de Veerdonk FL, Ferwerda G, Heinhuis B, Devesa I, et al: Differential requirement for the activation of the inflammasome for processing and release of IL-1beta in monocytes and macrophages. Blood 113: 2324-2335, 2009.

33. Strowig T, Henao-Mejia J,Elinav E and Flavell R: Inflammasomes in health and disease. Nature 481: 278-286, 2012. 